\title{
Therapeutic efficacy of an hTERT promoter-driven oncolytic adenovirus that expresses apoptin in gastric carcinoma
}

\author{
LEI LIU ${ }^{1,2,7}$, WENBIN WU ${ }^{2}$, GUANGZE ZHU ${ }^{2,3}$, LIMING LIU ${ }^{2,4}$, GUOFANG GUAN ${ }^{2,5}$, \\ XIAO LI ${ }^{1,2,6}$, NINGYI JIN ${ }^{2 *}$ and BAORONG CHI ${ }^{1 *}$ \\ ${ }^{1}$ Department of Gastroenterology, The First Hospital of Jilin University, Changchun 130021; \\ ${ }^{2}$ Genetic Engineering Laboratory of PLA, Academy of Military Medical Sciences of PLA, Changchun 130122; \\ ${ }^{3}$ Clinical Laboratory, The Affiliated Hospital to Changchun University of Chinese Medicine, Changchun 130021; \\ ${ }^{4}$ Jilin Academy of Agricultural Science and Technology, Jilin 132101; ${ }^{5}$ Head and Neck Surgery, \\ The Second Hospital of Jilin University, Changchun 130001; ${ }^{6}$ The Key Laboratory of Pathobiology, \\ Ministry of Education, Changchun 130021, P.R. China
}

Received March 27, 2012; Accepted May 7, 2012

DOI: 10.3892/ijmm.2012.1077

\begin{abstract}
The efficacy and specificity of treatment are the major challenges for cancer gene therapy. Oncolytic virotherapy is an attractive drug delivery platform of cancer gene therapy. Previous studies have determined that apoptin is a p53-independent, Bcl-2-insensitive apoptotic protein that has the ability to induce apoptosis specifically in tumor cells. In this study, we show that the administration of a dual cancer-specific oncolytic adenovirus construct, Ad-hTERT-E1a-apoptin [in which the adenovirus early region la (E1a) gene is driven by the cancer-specific promoter of human telomerase reverse transcriptase (hTERT) and that expresses apoptin simultaneously], suppresses tumor growth in gastric carcinoma cells in vitro and reduces the tumor burden in vivo in xenografted nude mice. The observation that infection with the Ad-hTERT-Ela-apoptin construct significantly inhibited the growth of gastric cancer cells and protected normal human gastric epithelium from growth inhibition confirmed the induction of cancer cell-selective adenovirus replication, growth inhibition and apoptosis by this therapeutic approach. In vivo assays were performed using BALB/c nude mice that had established primary tumors. Subcutaneous primary tumor volume was reduced not only in the intratumoral injection group but also in the systemic delivery mice following treatment with Ad-hTERT-
\end{abstract}

Correspondence to: Dr Xiao Li, The Key Laboratory of Pathobiology, Ministry of Education, 126 Xinmin St., Changchun 130021, P.R. China

E-mail: lixiao06@mails.jlu.edu.cn

Present address: ${ }^{7}$ The First College of Clinical Medical Science, China Three Gorges University (Yichang Central People's Hospital), Yichang 443003, P.R. China

\section{*Senior authorship}

Key words: antitumor, apoptin, apoptosis, conditional replicationcompetent adenovirus, gastric carcinoma
E1a-apoptin. Furthermore, treatment of primary models with Ad-hTERT-Ela-apoptin increased the mouse survival time. These data reinforce previous research and highlight the potential therapeutic application of Ad-hTERT-Ela-apoptin for the treatment of neoplastic diseases in clinical trials.

\section{Introduction}

Oncolytic virotherapy is a promising approach for patients who are resistant to traditional cancer therapies. A number of strategies have been evaluated in pre-clinical and clinical trials (1-3), including treatment with conditional replicationcompetent adenovirus (CRCA) (4-6). However, insufficient efficacy and poor specificity of treatment remain the major challenges for this targeted cancer gene therapy $(4,5)$ and highlight the need for the development of novel cancer therapies.

The human telomerase reverse transcriptase (hTERT) promoter is highly activated in immortalized cell lines and in over $85 \%$ of human cancers and has therefore been used widely for the construction of CRCAs $(4,7)$. In these studies, early region 1a (E1a) gene, which plays a central role in viral infection by regulation of viral replication and the cell cycle, was the gene used most frequently in the constructs and was essential for adenovirus replication $(8,9)$.

Apoptin, a protein of $13.6 \mathrm{kDa}$ in mass that was derived from the chicken anemia virus (CAV) VP3 gene and is thought to induce apoptosis selectively in a variety of tumor cells, such as hepatomas, lymphomas, cholangiocarcinomas, melanomas, as well as breast, lung, oral and colon carcinomas (10-12). Preliminary studies have demonstrated that more than 70 different transformed and malignant cells of human origin are sensitive to apoptin-induced apoptosis (11). However, apoptin does not act on normal non-transformed human cells, such as primary fibroblasts, smooth muscle cells, T cells, hepatocytes, hematopoietic stem cells, keratinocytes, or endothelial cells $(10,13,14)$. Furthermore, the long-term expression of apoptin in normal human fibroblasts has shown that it has no toxic or transforming activity in these cells $(11,15)$. All these 
factors make apoptin an attractive candidate for cancer gene therapy and numerous studies have demonstrated the effects of apoptin inserted into various vectors on the restriction of manifold tumors $(4,16,17)$.

In the present study, we show the significant antitumor activity of a novel dual cancer-specific oncolytic adenovirus construct, designated as Ad-hTERT-Ela-apoptin (4), which consists of a cancer-specific promoter (hTERT promoter) and a cancer cellselective apoptosis-inducing gene (apoptin). We found that the Ad-hTERT-E1a-apoptin construct exhibited selective replication and specific induction of apoptosis in human gastric cancer cells. Furthermore, we also show that Ad-hTERT-Ela-apoptin induces apoptosis in human gastric cancer cells specifically and induces apoptosis more rapidly when compared with the control viruses. These results suggest that the Ad-hTERT-E1a-apoptin construct is a potentially applicable anti-cancer agent for the treatment of human gastric carcinoma and may have clinical use in other neoplastic diseases.

\section{Materials and methods}

Cell lines, viruses and animals. The human gastric cancer cells, SGC7901, the human gastric epithelial cells, GES-1, and the human embryonic kidney cells, HEK-293, were obtained from the Type Culture Collection of the Chinese Academy of Sciences (Shanghai, China). The cells were maintained in Dulbecco's modified Eagle's medium (DMEM; HyClone, Beijing, China) that contained $10 \%$ heat-inactivated fetal bovine serum (FBS; HyClone), penicillin (100 U/ml) and streptomycin $(100 \mathrm{mg} / \mathrm{ml})$ at $37^{\circ} \mathrm{C}$ under an atmosphere of $95 \%$ air and $5 \% \mathrm{CO}_{2}$. All cell lines were passaged for no more than 6 months after receipt. The cells were routinely subcultured every 2-3 days, and were all taken from the logarithmic phase of growth. Female BALB/c nude mice (nu/nu, 6-8-week-old) were purchased from the Experimental Animal Center of the Academy of Military Medical Sciences (Beijing, China) and were housed in a pathogen-free facility for the duration of all experiments, following institute guidelines.

Recombinant adenoviruses. The construction and characterization of the dual cancer-specific oncolytic adenovirus, Ad-hTERT-Ela-apoptin, and the control viruses, Ad-mock, Ad-CMV-apoptin, Ad-hTERT-apoptin, Ad-CMV-E1a, Ad-hTERT-Ela and Ad-CMV-Ela-apoptin, used in this study have been described previously (4). Briefly, shuttle plasmids were constructed to generate the recombinant adenoviruses (Fig. 1). Packaging and production of the recombinant adenoviruses were performed in HEK-293 cells. Purification and titration of the virus stock were performed using the Adeno-X Virus Purification kit and Adeno-X Rapid titer kit (both from BD Biosciences Clontech).

MTT viability assay. Cell viability was determined by standard 3-(4,5-dimethylthiazol-2-yl)-2,5-diphenyl tetrazolium bromide (MTT; Sigma, St. Louis, MO, USA) assays as described previously $(4,18)$. In brief, cells were seeded in 96-well plates $\left(1 \times 10^{4}\right.$ cells/well $)$ and 1 day later the cells were infected with various concentrations $[1$ multiplicity of infection (MOI), 10 and $100 \mathrm{MOI}$ ] of the recombinant adenoviruses. Cell viability was measured every day over a 4-day period.

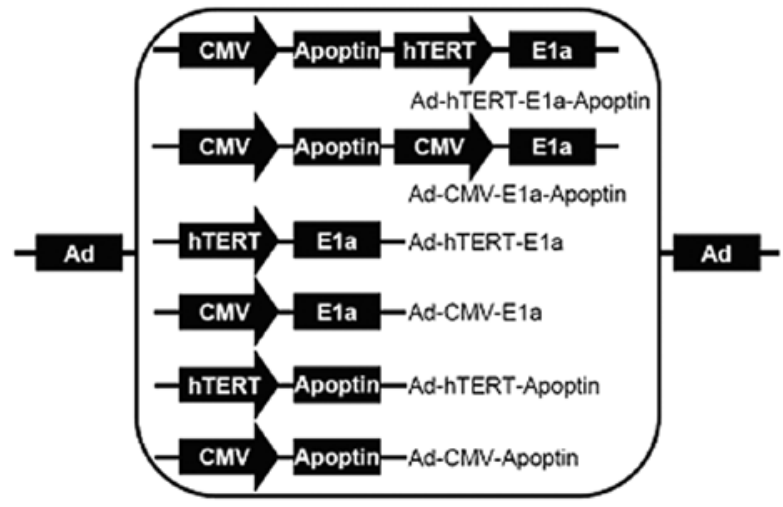

Figure 1. Schematic diagram of organization elements in recombinant adenoviruses. In the dual cancer-specific oncolytic adenovirus (Ad-hTERTEla-apoptin), the hTERT core promoter (the 5 ' flanking region of the hTERT gene between positions -283 to -78) drives Ela and the cytomegalovirus (CMV) promoter to drive apoptin expression. In cancer-specific replication-competent adenoviruses (Ad-hTERT-Ela), the hTERT promoter drives Ela. In non-specific replication-competent adenoviruses (Ad-CMV-Ela and Ad-CMV-Ela-apoptin), the CMV promoter drives Ela. Ad-hTERT-apoptin and Ad-CMV-apoptin have no replication activity in cancer and normal cells.

The percentage of cell death was expressed with respect to the control values using the following formula: [100x (control cells - experimental cells)/(control cells)] (4). Untreated cells were used as the controls and all measurements were repeated in triplicate.

Acridine orange (AO)/ethidium bromide (EB) staining assay. The AO/EB staining assay was performed as described previously to determine the relative percentage of live, necrotic and apoptotic cells $(16,19)$. Cells that had been infected with the recombinant adenoviruses were trypsinized and washed 3 times in Hank's balanced salt solution (HBSS). A 250- $\mu 1$ aliquot that contained $1 \times 10^{6}$ cells was incubated with $2 \mu \mathrm{l}$ of EB and $2 \mu \mathrm{l}$ of AO. The cells were then observed under a fluorescence microscope (BX51) and the images of representative cells were captured with a charged-coupled device (CCD) camera (DP71) (all were from Olympus). Samples were processed simultaneously, and all images were captured using the same parameters. Tests were performed in triplicate and at least 500 cells were measured from each sample to determine the presence of normal, apoptotic, or necrotic chromatin.

Western blot analysis. Cells were infected with the recombinant adenoviruses; target proteins were analyzed by western blot analysis as described previously (4). All antibodies were purchased from Santa Cruz Biotechnology, Inc. (Santa Cruz, CA, USA). Protein bands were visualized with the Pierce ECL Western Blotting Substrate (Pierce, Shanghai, China). Extracts of Ad-mock-infected cells were used as the negative control; detection of glyceraldehyde 3-phosphate dehydrogenase (GAPDH) was used as the internal control.

Tumor xenograft experiments. In vivo antitumor experiments were performed in 2 independent models. Briefly, $1 \times 10^{6}$ SGC7901 cells were implanted subcutaneously into the right flanks of BALB/c nude mice. Two weeks later, after establishment of the tumors, the tumor-bearing mice were divided randomly into 8 groups ( 5 mice/group). The mice in the first 
A

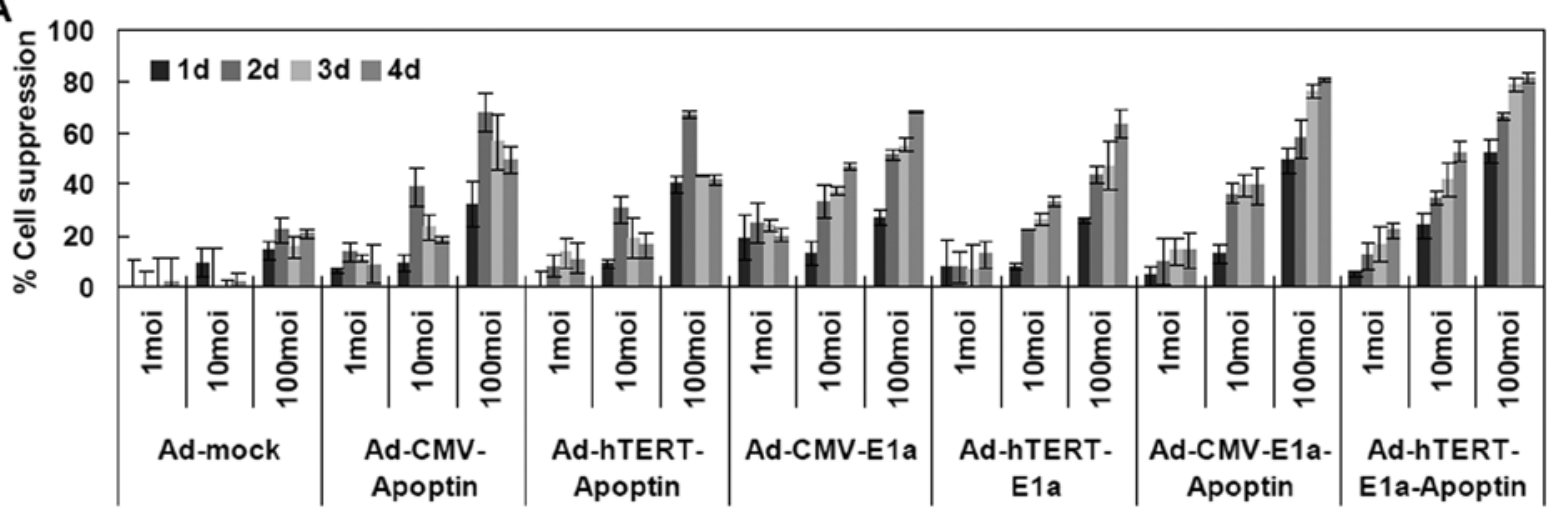

B

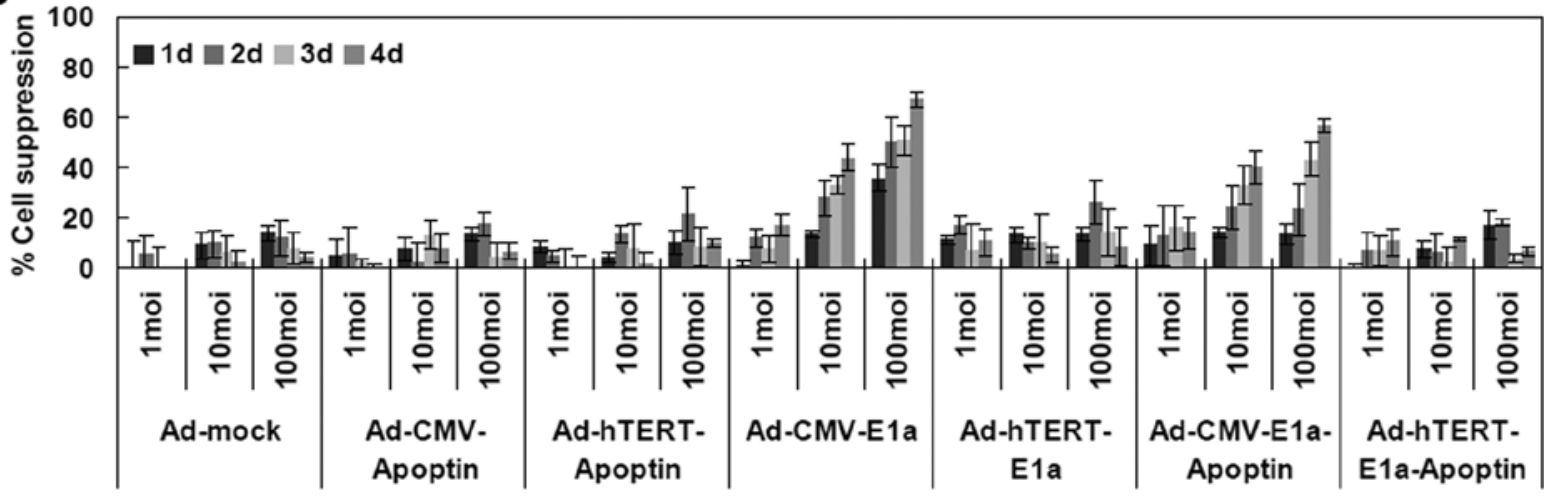

Figure 2. Assessment of the selective inhibitory effect of Ad-hTERT-E1a-apoptin on gastric cancer cells. Effects of the different multiplicities of infection (MOIs) and infection times on (A) SGC7901 cell viability; and (B) GES-1 cell viability. Cells were seeded into 96-well plates (1x10 cells/well) 1 day before cells were infected with various concentrations $(1,10$ and $100 \mathrm{MOI})$ of the indicated adenoviruses. Tumor viability was measured every day over a 4-day period by 3-(4,5-dimethylthiazol-2-yl)-2,5-diphenyl tetrazolium bromide (MTT) colorimetric assay and all measurements were performed in triplicate. Data are presented as the means \pm standard deviation (SD). (A) In SGC7901 cells, infection with Ad-hTERT-Ela-apoptin, Ad-hTERT-E1a-apoptin, Ad-CMV-E1a and Ad-hTERT-E1a induced growth inhibition. (B) By contrast, in GES-1 cells, infection with Ad-CMV-E1a or Ad-CMV-E1a-apoptin, but not Ad-CMV-apoptin, Ad-hTERT-apoptin, Ad-hTERT-Ela or Ad-hTERT-Ela-apoptin resulted in significant growth inhibition.

model received intratumoral injections of various recombinant adenoviruses at a dose of $1 \times 10^{9}$ plaque-forming units (pfu) in $50 \mu \mathrm{l}$ of saline, and the control group received $50 \mu \mathrm{l}$ of saline alone. In the second model, the injections were administered via the tail vein. All injections were given every 2 days for the first week (days 12, 14 and 16 after implantation) and once weekly for 2 more weeks (days 23 and 30 after implantation). Tumor size was measured using calipers twice a week and calculated with a formula of $\left[0.52\right.$ (smallest diameter) ${ }^{2} \mathrm{x}$ (largest diameter)] $(4,16,17)$. During the tumor study, all animals were monitored daily and sacrificed at the end of the experiment.

Statistical analysis. Statistical significance of the results was calculated using one-way analysis of variance (ANOVA) and results were deemed to be statistical significant if the P-value was $<0.05$. Log-rank tests were used to calculate survival rates. Data from all animals are represented as Kaplan-Meier plots.

\section{Results}

Selectively inhibitory effects of Ad-hTERT-Ela-apoptin on gastric cancer cells. The correlation between infection time and MOI was complex and synergistic and consistent with the results of previous studies $(4,16)$ (Fig. 2). At longer infection times, the growth rates of SCG7901 or GES-1 cells that were infected with replication-competent adenoviruses (Ad-CMV-E1a, Ad-hTERT-E1a, Ad-CMV-Ela-apoptin and Ad-hTERTEla-apoptin) were inhibited (Fig. 2A). However, cells treated with Ad-CMV-apoptin, Ad-hTERT-apoptin and Ad-mock gradually resumed their growth rate after 2 days (Fig. 2A). By contrast, the growth of GES-1 cells infected only with non-specific replication-competent adenoviruses (Ad-CMV-E1a or Ad-CMV-Ela-apoptin) was suppressed (Fig. 2B). Furthermore, cell viabilities were dependent to a certain extent on the MOI of the recombinant adenoviruses. In the SGC7901 human gastric cancer cells, infection with Ad-CMV-Ela-apoptin or Ad-hTERT-Ela-apoptin at a MOI of 1 or 10 induced a cell growth inhibition of $10-25$ or $35-55 \%$ after 4 days, respectively, and the growth of cells infected with 100 MOI was $80-85 \%$. When infected with 1 or $10 \mathrm{MOI}$ of Ad-CMV-Ela or Ad-hTERT-E1a, cell growth was inhibited by $30-50$ or $60-70 \%$ after 4 days, respectively. However, in the GES-1 human gastric epithelial cells, dose-dependent inhibition was only observed in cells in the Ad-CMV-E1a and Ad-CMV-E1a-apoptin experimental groups. Therefore replication-competent adenoviruses were much more potent than the replication-incompetent adenoviruses in gastric cancer cell suppression, and adenoviruses with the dual cancer-specific genes were more effective than the normal replication-incompetent adenoviruses. In addition, Ad-hTERT-Ela-apoptin induced 


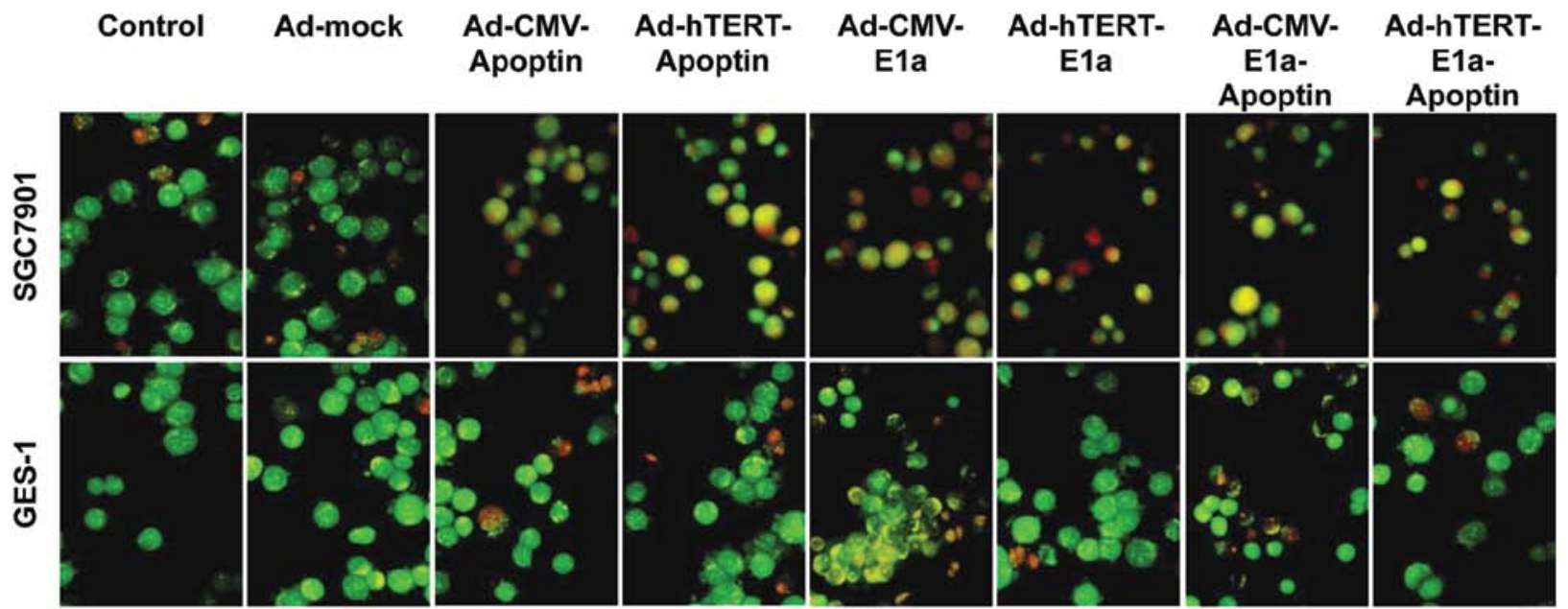

Figure 3. Morphological changes of SGC7901 and GES-1 cells infected with Ad-hTERT-E1a-apoptin by acridine orange/ethidium bromide (AO/EB) staining. (A) Fluorescence images of adenovirus-infected SGC-7901 or GES-1 cells stained with AO/EB. Representative images at x400 magnification were used to show AO/EB staining. Infection with Ad-CMV-E1a alone and Ad-CMV-E1a-apoptin elevated the percentage of apoptotic cells in normal GES-1 cells. However, all recombinant adenoviruses, except for Ad-mock, resulted in significant apoptosis in SGC-7901 cells. (B) Quantification of live, necrotic and apoptotic cells. Data are presented as the means \pm standard deviation (SD). The images from the microscope were captured with the Image-Pro Plus (version 5.0.2) software program and analyzed the following day. Tests were performed in triplicate and at least 500 cells were scored for each sample to determine the presence of normal, apoptotic or necrotic chromatin.

growth suppression selectively in cancer cells without harming the normal counterparts. That is, Ad-hTERT-Ela-apoptin replicated specifically in gastric cells (SGC7901) and restricted the cell growth selectively, exhibiting higher tumor-specific killing activity than the control viruses.

Ability of Ad-hTERT-Ela-apoptin to induce tumor-specific apoptosis. Using the AO/EB method, we compared and quantified the percentage of live, necrotic and apoptotic cells of the control and recombinant adenovirus-treated SGC7901 and GES-1 cells. Live cells have a normal green nucleus; early apoptotic cells have a bright green nucleus with condensed or fragmented chromatin; late apoptotic cells display condensed and fragmented orange chromatin; and cells that have died from direct necrosis have a structurally normal orange nucleus (20) (Fig. 3A). Infection with all recombinant adenoviruses resulted in apoptosis of SGC7901 cells, whereas, in GES-1 cells, only infection with Ad-CMV-Ela or Ad-CMV-E1a-apoptin was cytotoxic (Fig. 3A). Furthermore, the proportion of live, necrotic and apoptotic cell populations in the control and treated SGC7901 or GES-1 cells was significantly different (Fig. 3B). In SGC7901 cells, infection with adenoviruses that expressed apoptin predominantly caused apoptosis; however infection with Ad-CMV-E1a or Ad-hTERT-Ela mainly caused necrosis. In GES-1 cells, due to the loss of the apoptosis-inducing effect of apoptin, infection with Ad-CMV-Ela-apoptin mainly caused necrosis, similar to the Ad-CMV-Ela-infected cells. These results indicated that although the recombinant adenoviruses had a significant in vitro antitumor effect via the induction of necrosis, Ad-hTERT-E1a-apoptin significantly restrained the growth of SGC7901 cells by the induction of apoptosis.

Effects of Ad-hTERT-E1a-apoptin on caspases in SGC7901 cells. The activation of caspases and corresponding markers detected by antibodies specific for the activated form of these proteins was determined by western blot analysis. Infection of SGC7901 cells with the recombinant adenoviruses caused a marked increase in the active cleaved subunit of caspase- 3 (Fig. 4A). Furthermore, the recombinant adenovirus infection resulted in detectable expression of cleaved lamin A, a marker for caspase- 6 activation. Cleaved poly ADP-ribose polymerase (PARP) was also detected in the infected SGC7901 cells, which not only indicated the activation of caspase-7 but also reconfirmed the existence of an active caspase-3. By contrast, the activation of the caspases and the corresponding substrates was detected at only a limited degree in GES-1 cells infected with the recombinant adenoviruses except for Ad-CMV-E1a and Ad-CMV-E1a-apoptin (Fig. 4B). These results suggested that the specific activation of caspases-3, -6 and -7 in SGC7901 cells was associated with apoptin expression and viral replication.

Growth tendency and life span analysis in vivo. For ethical reasons, all animals in the study were sacrificed by day 63 before the tumors grew to a volume of $2,500 \mathrm{~mm}^{3}$. Therefore, only the mean survival curve of each group of mice was obtained. When the injections were performed intratumorally, the saline-treated and Ad-mock-infected groups had the worst survival rate, while infection with the recombinant adenoviruses significantly improved the survival time (Fig. 5A). The mean survival times were 47.5 days for the saline-treated nude mice, 41.5 days for the Ad-mock-infected nude mice and 63 days for mice infected with the other recombinant adenoviruses. This observation demonstrates that the intratumoral injection of apoptin-expressing recombinant adenoviruses effectively improves survival. In the second model, the intravenous injection of Ad-hTERT-Ela-apoptin or Ad-CMV-Ela-apoptin significantly increased the survival of nude mice in comparison with the other recombinant adenovirus-infected or 

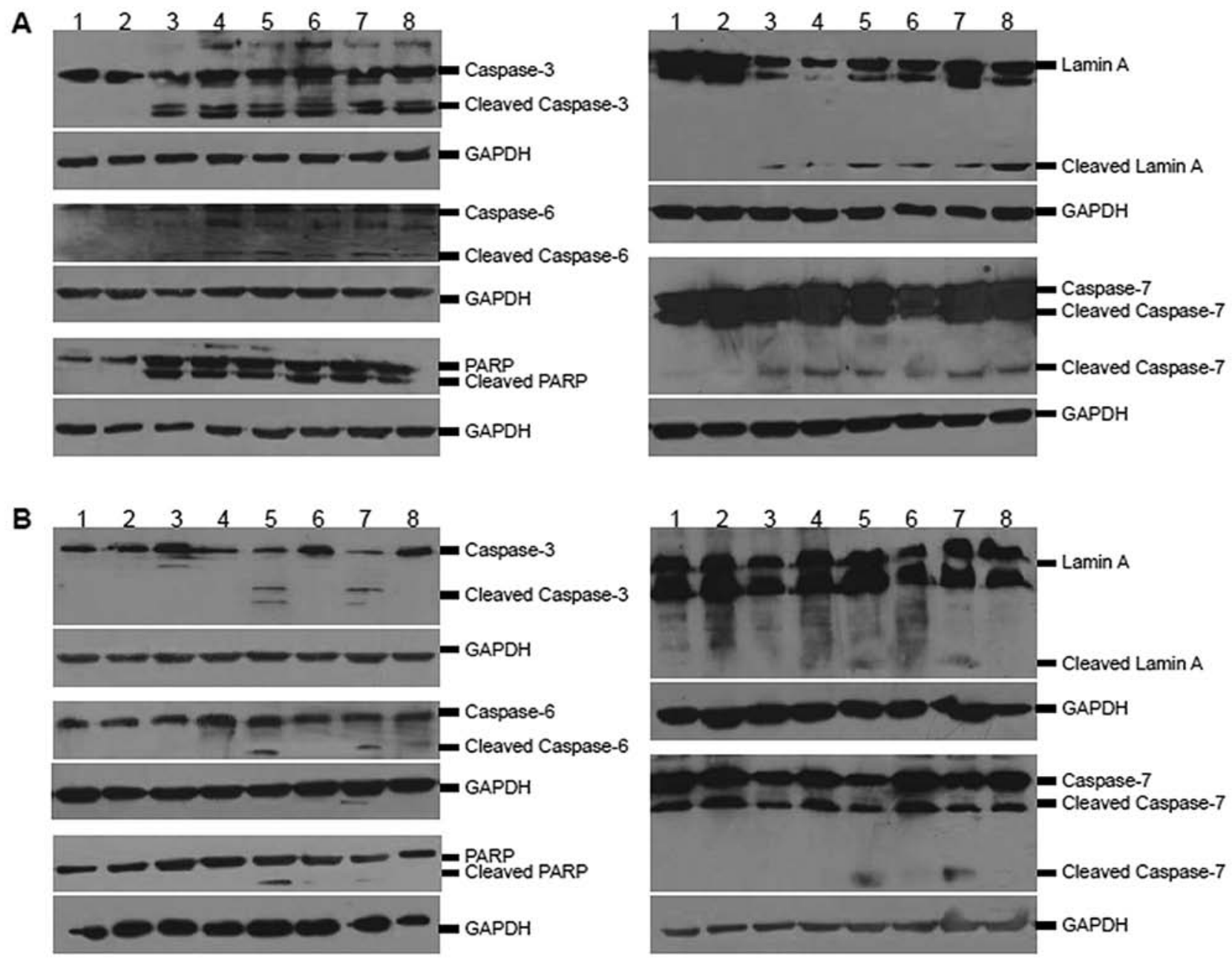

Figure 4. Western blot analysis detection of caspase activation in recombinant adenovirus-treated cells. (A) SGC-7901 cells; and (B) GES-1 cells were treated with the indicated recombinant adenoviruses for $48 \mathrm{~h}$ and subjected to western blot analysis; the corresponding bands that represented caspases or their substrates are indicated. GAPDH was used to normalize the results for total proteins. Lane 1, control; lane 2, Ad-mock; lane 3, Ad-CMV-apoptin; lane 4, Ad-hTERT-apoptin; lane 5, Ad-CMV-Ela; lane 6, Ad-hTERT-E1a; lane 7, Ad-CMV-E1a-apoptin; and lane 8, Ad-hTERT-Ela-apoptin.

saline-treated mice (Fig. 5B). At the end of the experiment, the survival rates of the treated mice were $0 \%$ (saline), $0 \%$ (Ad-mock), 80\% (Ad-CMV-apoptin), 40\% (Ad-hTERTapoptin), 60\% (Ad-CMV-E1A), 40\% (Ad-hTERT-E1a), 100\% (Ad-CMV-Ela-apoptin) and 100\% (Ad-hTERT-Ela-apoptin). The mean survival times of the groups that received an injection in the tail vein were 40.5 days for the saline-treated mice, 42.5 days for the Ad-mock-infected mice, 58.5 days for the Ad-CMV-apoptin-infected mice, 52.5 days for the Ad-hTERTapoptin-infected mice, 53.5 days for the Ad-CMV-Ela-infected mice and 55.5 days for the Ad-hTERT-E1a-infected mice. These results indicate that the intravenous injection of the dual cancer-specific oncolytic adenovirus confers significant survival benefits in vivo.

We also evaluated the growth kinetics of the tumors following treatment. The tumors in the recombinant adenovirus groups were suppressed after the infection in the first model, compared with the control and Ad-mock-infected groups (Fig. 5C). However, the Ad-CMV-Ela- and Ad-hTERTEla-infected tumors gradually resumed their growth at 6 weeks after the first injection, whereas the growth of the majority of the Ad-CMV-E1a-apoptin-infected or Ad-hTERTEla-apoptin-infected tumors was markedly reduced. These results indicate that the intratumoral injection of Ad-CMVEla-apoptin or Ad-hTERT-E1a-apoptin induces a powerful antitumor response. In the systemic delivery model (Fig. 5D), the tumors infected with Ad-CMV-Ela-apoptin or Ad-hTERTEla-apoptin began to show growth impairment after 2 weeks, consistent with the results of the intratumoral injection experiment. Significant differences between the other groups and Ad-CMV-E1a-apoptin or the Ad-hTERT-E1a-apoptin groups were confirmed during follow-up. The growth of the Ad-CMV-apoptin-, Ad-hTERT-apoptin-, Ad-CMV-E1a- or Ad-hTERT-E1a-infected tumors was suppressed compared with the saline-treated and Ad-mock infected mice; however, the differences in size impairment of the tumors in these groups were slight. These results demonstrate that the systemic delivery of Ad-hTERT-E1a-apoptin or Ad-CMV-E1a-apoptin significantly reduces tumor burdens and that the dual cancerspecific oncolytic adenoviruses are a much more potent tool than replication-incompetent or non-specific replicationcompetent adenoviruses for cancer cell suppression. 

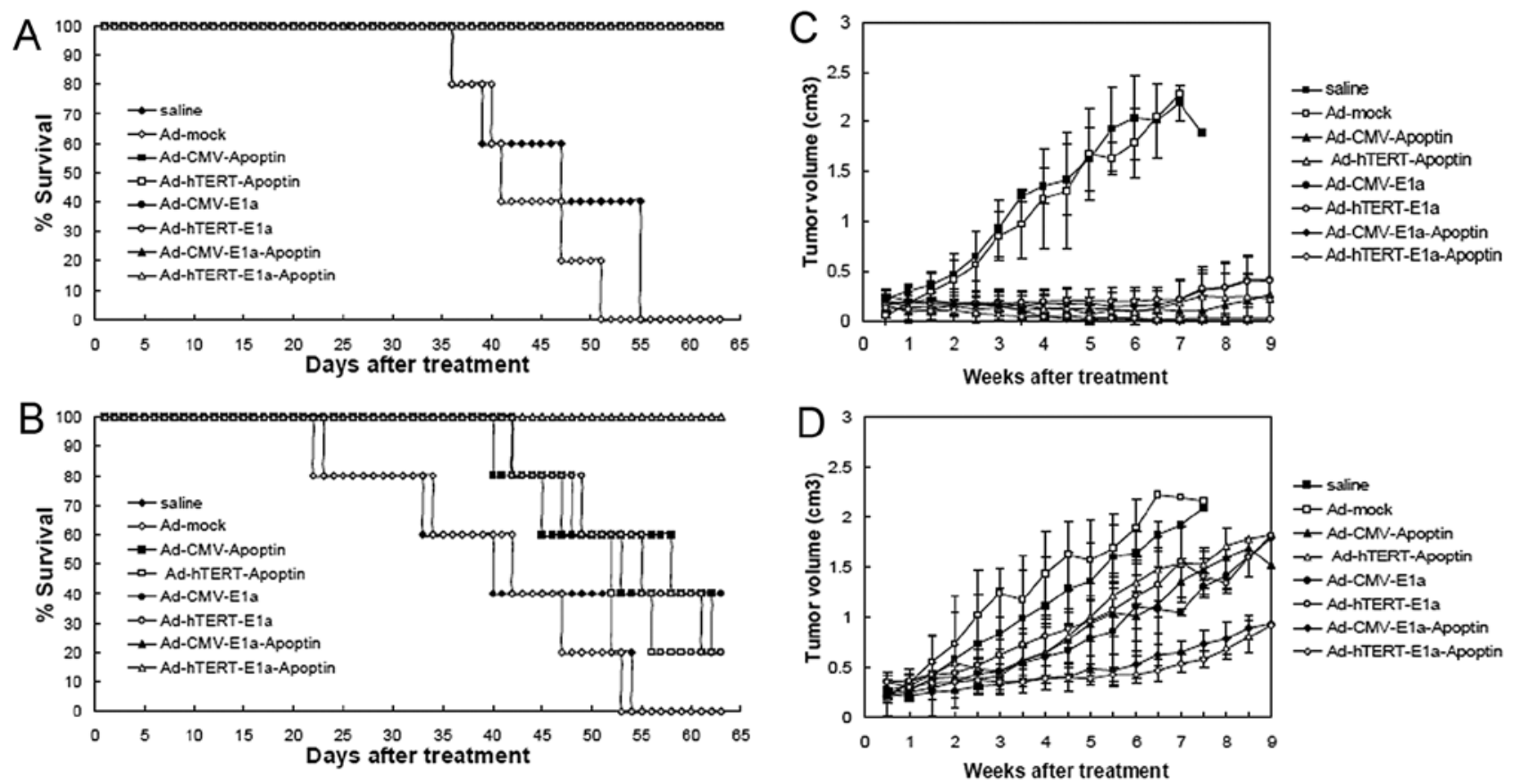

Figure 5. Ad-hTERT-E1A-apoptin suppression of gastric cancer in the BALB/c nude mice model. (A) Survival curve of mice treated intratumorally. (B) Survival curve of mice treated intravenously. (C) Tumor growth kinetics of mice that received injections intratumorally. (D) Tumor growth kinetics of mice that received injections intravenously. The day that the first injection performed was considered to be day 0 . (C and D) Data are represented as the means \pm standard deviation (SD). (A and B) Increased mean survival was also observed in Ad-CMV-E1a-apoptin- or Ad-hTERT-E1a-apoptin-treated mice in comparison with the saline or Ad-mock-treated mice in the tumor models. Furthermore, Ad-CMV-Ela-apoptin or Ad-hTERT-E1a-apoptin significantly inhibited the growth of tumors in the (C) intratumoral and (D) systemic delivery groups. (D) Although Ad-CMV-apoptin, Ad-hTERT-apoptin, Ad-CMV-Ela or Ad-hTERT-Ela had some inhibitory effect on tumors in systemic delivery groups, the antitumor effects were less potent.

\section{Discussion}

Gastric cancer is a worldwide health burden. Treatment has limited progress and $75 \%$ of patients are diagnosed only at the unresectable stage (21). Following curative resection alone or even after adjuvant therapy, approximately $60 \%$ of affected patients succumb to gastric cancer (22). All these factors pose the challenge of whether to choose a strictly supportive approach or to expose patients to the side-effects of a potentially ineffective treatment.

Cancer gene therapy based on adenoviruses has been studied extensively in pre-clinical and clinical trials $(2,3)$. In particular, use of CRCAs has gained increased attention for a number of reasons $(5,23)$. Importantly, as the promoters in these vectors are selective for cancer cells $(5,23)$, these oncolytic viruses have the ability to replicate and spread to adjacent tumor cells (24). Furthermore, infection with CRCA generates antitumoral immune responses (25) that can complement chemotherapy and radiotherapy (24). Additionally, CRCAs are capable of achieving the destruction of primary and distant tumors given the proper therapeutic transgenes (5).

In the present study, we describe the antitumor effects of a dual cancer-specific oncolytic adenovirus, Ad-hTERTEla-apoptin, in which replication was driven by the hTERT promoter that replicates selectively and induces apoptosis specifically in tumor cells. The antitumor effects were evident within $24 \mathrm{~h}$ when administered to the gastric cancer cells in vitro. Treatment with 100 or 10 MOI doses completely inhibited the growth of SGC7901 cells 4 days later (Fig. 2A), although a single Ad-hTERT-Ela-apoptin treatment at $1 \mathrm{MOI}$ was less effective. By contrast, growth inhibition was not observed in GES-1 cells after treatment with Ad-hTERT-E1aapoptin at any MOI dose (Fig. 2B). These findings indicate that Ad-hTERT-E1a-apoptin replicates specifically and induces growth suppression selectively in SGC7901 cells compared with the normal counterparts.

The applicability of cancer therapies is not only determined by their efficiency as specificity is an equally important prerequisite (26). Various studies have indicated that more than 70 tumor cell lines are susceptible to apoptin, whereas apoptin does not affect a variety of normal and non-transformed cells $(13,27)$. In contrast to our findings, Guelen et al (28) showed the toxicity of apoptin towards non-cancerous cells; however, this study demonstrated cell death only in a fetal cell type. Furthermore, the ability of apoptin to induce tumorspecific apoptosis is independent of p53 $(13,29)$. Thus, apoptin is similarly effective at killing tumor cells that are p53-deficient or that express either wild-type or mutant p53 $(13,29)$. The role of anti-apoptotic molecules in apoptin-induced apoptosis is still a matter of debate. In certain tumor cell lines, apoptin-mediated cell death is independent of the Bcl-2 status and is even stimulated by Bcl-2 or is insensitive to Bcr-Abl and Bcl-xL $(13,30)$. Based on these concepts, apoptin can be used to complement radiotherapeutic and chemotherapeutic approaches.

Chromatin condensation and nuclear fragmentation remain the hallmarks of apoptotic cells $(16,20)$. Fluorescence light microscopy with differential uptake of fluorescent DNA-binding dyes (such as EB/AO staining) is the method of choice due to its 
simplicity. In this study, using the AO/EB method, we quantified the percentage of live, necrotic and apoptotic cells after recombinant adenovirus treatment (Fig. 3). The infection of SGC7901 cells with the recombinant adenoviruses may, in part, inhibit the cells by causing necrosis. We expected, therefore, that the effects of the apoptin gene may be masked. However, the effects of the recombinant virus did not impede apoptininduced apoptosis. After Ad-hTERT-Ela-apoptin infection, $38.6 \%$ of apoptin-expressing transformed cells became apoptotic after only $48 \mathrm{~h}$, and $27.1 \%$ of infected cells exhibited necrosis. By contrast, although $24.3 \%$ of cells were apoptotic $48 \mathrm{~h}$ after Ad-hTERT-E1a infection, $25.5 \%$ of cells died as a result of necrosis. These results indicate that, although replication-component adenovirus infection stimulates necrosis, this event does not appear to render tumor cells sensitive to apoptin. Furthermore, classification of cell death should always include morphological examination coupled with at least one other assay. Apoptin induces apoptosis in a wide variety of human cancer cell lines via the classical apoptotic pathways (31). Caspases-3, -6 and -7 are the important downstream effecter caspases that cleave major cellular substrates in apoptotic cells and amplify these substrates via intrinsic or extrinsic pathways (32). In the present study, a western blot analysis of total protein extracts showed activation of caspases-3, -6 and-7 in recombinant virusinfected SGC7901 cells. However, no caspase activation was observed in GES-1 cells, except for non-specific replicationcompetent adenovirus infection. GAPDH expression was used to confirm equal loading of the gel. These results validate the specific apoptosis-inducing effects of the dual cancer-specific oncolytic adenovirus Ad-hTERT-Ela-apoptin construct.

We also evaluated antitumor activity in vivo in a human gastric cancer xenograft mouse model, which confirmed and extended the results of the in vitro studies. In this study, tumors derived from mouse gastric cancer cells transplanted into $\mathrm{BALB} / \mathrm{c}$ nude mice were infected with various recombinant adenoviruses. We found that all recombinant adenoviruses had a significant antitumor effect, despite the fact that the replication-defective adenoviruses infected only part of the tumors. The injection of Ad-hTERT-Ela-apoptin resulted in a complete response to treatment (Fig. 5). However, infection with Ad-hTERT-apoptin, Ad-CMV-apoptin, Ad-hTERT-E1a or Ad-CMV-E1a was less effective. It is plausible that the application of replication-component adenoviruses allows virus dispersion into any tumor tissues in the animal and that apoptin expression enhances the capability of the oncolytic adenovirus. When infection was carried out intravenously, however, treatment with Ad-hTERT-Ela-apoptin did not lead to complete tumor regression, although the tumor growth was significantly delayed (Fig. 5). The suppression of the indirectly injected tumor may also reflect a secondary viral infection by the CRCA. The oncolytic activity of Ad-hTERT-apoptin, Ad-CMV-apoptin, Ad-hTERT-Ela or Ad-CMV-Ela was limited, which was similar to that of intratumoral injection groups. No tumor regression was observed in mice that had SGC7901 tumors when injected intravenously with saline and Ad-mock. Furthermore, we did not observe any toxic effects after injection of Ad-hTERT-Elaapoptin in the in vivo experiments described in this study. Thus, our data indicate that there is great potential for improvement in the safety and efficacy of adenovirus vectors for wide application in the treatment of neoplastic diseases.
In conclusion, gene therapy with apoptin offers unique advantages over current approaches for cancer therapy. Factors such as: i) apoptin does not need a functional p53 pathway; ii) it is not hindered by the commonly found blockage of apoptosis by Bcl-2 or Bcr-Abl, iii) it acts downstream of most other factors, and iv) it has unparalleled potency, suggest that apoptin is applicable for the treatment of a wide range of tumors. In addition, the CRCA we describe in this study selectively induces apoptosis in various cancer cells without adverse effects on normal cells. In vivo and in vitro data described in this study show that the expression of apoptin increases the effectiveness of CRCAs and that an adenovirus vector under the control of a hTERT promoter does not reduce the efficacy of the construct but improves the global safety of CRCAs. All these factors highlight the need for further evaluation of this strategy for clinical trials.

\section{Acknowledgements}

This study was supported in part by grants from the National Science and Technology Major Projects for 'Major New Drugs Innovation and Development' (no. 2010ZX09401-305-14), the National Natural Science Foundation of China (nos. 81072210 and 81101140), the Key Technologies R\&D Programme of Jilin Province (nos. 10ZDGG007 and 201015166) and the China Postdoctoral Science Foundation (no. 20100481057).

\section{References}

1. Xu Y, Liu Z, Kong H, et al: Co-expression of interleukin 12 enhances antitumor effects of a novel chimeric promoter-mediated suicide gene therapy in an immunocompetent mouse model. Biochem Biophys Res Commun 412: 763-768, 2011.

2. Alemany R: Cancer selective adenoviruses. Mol Aspects Med 28: 42-58, 2007.

3. Haviv YS and Curiel DT: Conditional gene targeting for cancer gene therapy. Adv Drug Deliv Rev 53: 135-154, 2001.

4. Li X, Liu Y, Wen Z, et al: Potent antitumor effects of a dual specific oncolytic adenovirus expressing apoptin in vitro and in vivo. Mol Cancer 9: 10, 2010.

5. Sarkar D, Su ZZ, Vozhilla N, Park ES, Gupta P and Fisher PB: Dual cancer-specific targeting strategy cures primary and distant breast carcinomas in nude mice. Proc Natl Acad Sci USA 102: 14034-14039, 2005.

6. Post DE, Sandberg EM, Kyle MM, et al: Targeted cancer gene therapy using a hypoxia inducible factor dependent oncolytic adenovirus armed with interleukin-4. Cancer Res 67: 6872-6881, 2007.

7. Kovalenko OA, Kaplunov J, Herbig U, Detoledo S, Azzam EI and Santos JH: Expression of (NES-)hTERT in cancer cells delays cell cycle progression and increases sensitivity to genotoxic stress. PLoS One 5: e10812, 2010.

8. Wojciak JM, Martinez-Yamout MA, Dyson HJ and Wright PE: Structural basis for recruitment of $\mathrm{CBP} / \mathrm{p} 300$ coactivators by STAT1 and STAT2 transactivation domains. EMBO J 28: 948-958, 2009

9. Hoti N, Chowdhury WH, Mustafa S, et al: Armoring CRAds with p21/Waf-1 shRNAs: the next generation of oncolytic adenoviruses. Cancer Gene Ther 17: 585-597, 2010.

10. Danen-Van Oorschot AA, Fischer DF, Grimbergen JM, et al: Apoptin induces apoptosis in human transformed and malignant cells but not in normal cells. Proc Natl Acad Sci USA 94: 5843-5847, 1997.

11. Backendorf C, Visser AE, de Boer AG, et al: Apoptin: therapeutic potential of an early sensor of carcinogenic transformation. Annu Rev Pharmacol Toxicol 48: 143-169, 2008.

12. Schoop RA, Baatenburg de Jong RJ and Noteborn MH: Apoptin induces apoptosis in an oral cancer mouse model. Cancer Biol Ther 7: 1368-1373, 2008.

13. Los M, Panigrahi S, Rashedi I, et al: Apoptin, a tumor-selective killer. Biochim Biophys Acta 1793: 1335-1342, 2009. 
14. Rohn JL and Noteborn MH: The viral death effector Apoptin reveals tumor-specific processes. Apoptosis 9: 315-322, 2004.

15. Zhang YH, Leliveld SR, Kooistra K, et al: Recombinant Apoptin multimers kill tumor cells but are nontoxic and epitope-shielded in a normal-cell-specific fashion. Exp Cell Res 289: 36-46, 2003

16. Li X, Jin N, Mi Z, et al: Antitumor effects of a recombinant fowlpox virus expressing Apoptin in vivo and in vitro. Int $\mathbf{J}$ Cancer 119: 2948-2957, 2006.

17. Lian $\mathrm{H}$, Jin N, Li X, et al: Induction of an effective antitumor immune response and tumor regression by combined administration of IL-18 and Apoptin. Cancer Immunol Immunother 56: 181-192, 2007.

18. Li Q, Zhu J, Sun F, Liu L, Liu X and Yue Y: Oncostatin M promotes proliferation of ovarian cancer cells through signal transducer and activator of transcription 3. Int J Mol Med 28: 101-108, 2011.

19. Zainal Ariffin SH, Wan Omar WH, Zainal Ariffin Z, Safian MF, Senafi S and Megat Abdul Wahab R: Intrinsic anticarcinogenic effects of Piper sarmentosum ethanolic extract on a human hepatoma cell line. Cancer Cell Int 9: 6, 2009.

20. Candal E, Anadon R, DeGrip WJ and Rodriguez-Moldes I: Patterns of cell proliferation and cell death in the developing retina and optic tectum of the brown trout. Brain Res Dev Brain Res 154: 101-119, 2005.

21. Patrascu F, Croitoru A, Gramaticu I, Andrei M, Teiutanu A and Diculescu M: Locally advanced or metastatic gastric cancer - new therapeutic solutions. Rev Med Chir Soc Med Nat Iasi 115: 20-26, 2011 (In Romanian).

22. Milne AN, Carneiro F, O'Morain C and Offerhaus GJ: Nature meets nurture: molecular genetics of gastric cancer. Hum Genet 126: 615-628, 2009.
23. Wu CL, Shieh GS, Chang CC, et al: Tumor-selective replication of an oncolytic adenovirus carrying oct-3/4 response elements in murine metastatic bladder cancer models. Clin Cancer Res 14: $1228-1238,2008$

24. Kirn DH and McCormick F: Replicating viruses as selective cancer therapeutics. Mol Med Today 2: 519-527, 1996

25. Shisler J, Duerksen-Hughes P, Hermiston TM, Wold WS and Gooding LR: Induction of susceptibility to tumor necrosis factor by $\mathrm{E} 1 \mathrm{~A}$ is dependent on binding to either $\mathrm{p} 300$ or $\mathrm{p} 105-\mathrm{Rb}$ and induction of DNA synthesis. J Virol 70: 68-77, 1996.

26. Fischer U and Schulze-Osthoff K: New approaches and therapeutics targeting apoptosis in disease. Pharmacol Rev 57: 187-215, 2005.

27. Oro $C$ and Jans DA: The tumour specific pro-apoptotic factor apoptin (Vp3) from chicken anaemia virus. Curr Drug Targets 5: 179-190, 2004.

28. Guelen L, Paterson H, Gaken J, Meyers M, Farzaneh F and Tavassoli M: TAT-apoptin is efficiently delivered and induces apoptosis in cancer cells. Oncogene 23: 1153-1165, 2004.

29. Zhuang SM, Shvarts A, van Ormondt H, Jochemsen AG, van der Eb AJ and Noteborn MH: Apoptin, a protein derived from chicken anemia virus, induces p53-independent apoptosis in human osteosarcoma cells. Cancer Res 55: 486-489, 1995.

30. Noteborn MH: Proteins selectively killing tumor cells. Eur J Pharmacol 625: 165-173, 2009.

31. Pavet V, Portal MM, Moulin JC, Herbrecht R and Gronemeyer H: Towards novel paradigms for cancer therapy. Oncogene 30: 1-20, 2011.

32. Chowdhury I, Tharakan B and Bhat GK: Caspases - an update. Comp Biochem Physiol B Biochem Mol Biol 151: 10-27, 2008. 